\title{
A MATRIX REPRESENTATION FOR ASSOCIATIVE ALGEBRAS. II
}

\author{
BY
}

\section{JACQUES LEWIN $\left({ }^{1}\right)$}

ABSTRACT. The results of part I of this paper are applied to show that if $F$ is a $f$ ree algebra over the field $K$ and $W$ is a subset of $F$ which is algebraically independent modulo the commutator ideal $[F, F]$, then $W$ again generates a free algebra. On the way a similar theorem is proved for algebras that are free in the variety of $K$-algebras whose commutator ideal is nilpotent of class $n$.

It is al so shown that if $L$ is a Lie algebra with universal enveloping algebra $F$, and $U, V$ are ideals of $L$, then $F U F \cdot F V F \cap L=[U \cap V, U \cap V]$. This is used to extend the representation theorem of part $I$ to free Lie algebras.

I. Introduction. In this paper we give some further applications of the matrix representation theorem proved in part I [7]. We first prove a general result communicated to us by George Bergman: If $R$ is a ring and $l$ an ideal of $R$ which is free as a right $R$-module, and $x \in R$, then $x$ is a zero divisor in $R / l$ if and only if it is a zero divisor in $R / I^{n}$. Next, let $[F, F]$ be the commutator ideal of the free algebra $F$. The algebra $R=F /[F, F]^{n}$ is a free algebra in the variety (in the sense of universal algebra) of algebras whose commutator ideal is nilpotent of class at most $n$. We show that a subset $Z$ of $R$ freely generates a (relatively) free subalgebra if, and only if, $Z$ is algebraically independent modulo $[R, R]$. Since $\bigcap_{i=1}^{\infty}[F, F]^{i}=0$, this allows us to show that a subset of $F$ which is algebraically independent modulo $[F, F]$ generates a free subalgebra of $F$. In another direction, if $U, V$ are ideals of a Lie algebra $L$, and $U_{F}, V_{F}$ are the corresponding ideals in the universal associative enveloping algebra $F$, we show that $U_{F} V_{F} \cap L=[U \cap V, U \cap V]$. If $L$ is a free Lie algebra, the matrix representation [4] for $F$ with kernel $U_{F} V_{F}$ then gives a representation for $L$ with kernel $[U \cap V, U \cap V]$.

The author would like to express his appreciation to George Bergman who read two versions of this paper and suggested a number of improvements. In particular, the result of the next section, in this generality, is due to him.

II. Regular elements. The results of this section are due to G. Bergman.

Received by the editors March 2, 1972.

AMS (MOS) subject classifications (1970). Primary 16A06, 16A38, $17 \mathrm{~B} 35$.

Key words and phrases. Free algebras, free subalgebras, PI algebras, free Lie algebras.

(1) This work was supported by NSF grants GP-8094 and GP-33050. 
Lemma 1. Let $R$ be a ring, $f: M \rightarrow N$ a bomomorpbism of right $R$-modules, $f^{\prime}: M^{\prime} \rightarrow N^{\prime}$ its restriction to an appropriate pair of submodules, and $J$ a left ideal of $R$. Suppose that the induced maps $M / M^{0} \rightarrow N / N^{\prime}$ and $M / M J \rightarrow N / N J$ are injective, and that $N$ and $J$ are flat as (right, resp. left) R-modules. Then the induced map $M / M^{\prime} J \rightarrow N / N^{\prime} J$ is also injective

Proof. Injectivity of $M / M^{\prime} \rightarrow N / N^{\prime}$ and flatness of $J$ give the injectivity of the top arrow in the commutative diagram:

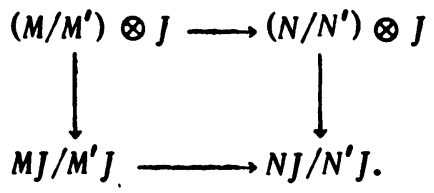

Here to construct the left-hand descending arrow, we tensor $M^{\prime} \rightarrow M \rightarrow M / M^{\prime}$ $\rightarrow 0$ with $J$ and conclude that $\left(M / M^{\prime}\right) \otimes J \simeq(M \otimes J) / \operatorname{Im}\left(M^{\prime} \otimes J\right)$, then use the natural map $M \otimes J \rightarrow M J$ to induce a map $M \otimes J / I m\left(M^{0} \otimes J\right) \otimes M J / M^{\prime} J$. The right-hand map is similarly obtained, but because $N$ is flat, the map $N \otimes J \rightarrow N J$ is an isomorphism, hence so is $N \otimes J / I m\left(N^{0} \otimes J\right) \rightarrow N J / N^{0} J$, and we see that the right-hand descending map is an isomorphism. As the top arrow is $1-1$, so is the diagonal composite map, hence so is the left-hand descending map. As the latter is onto, it too is an isomorphism; hence the bottom map is injective.

Combining this with the injectivity of $M / M J \rightarrow N / N J$, we get the desired injectivity of $M / M^{0} J \rightarrow N / N^{\prime} J$.

Lemma 2. Let $R$ be a ring (with 1), $M$ a generator in the category of left $R \cdot$-modules, and $J$ a two-sided ideal of $R$. If $x \in R$ acts as a left-zero-divisor on $R / J$, then it acts as a zero-divisor on $M / M J$.

Proof. Since $M$ is a generator, some direct $\operatorname{sum} N=M \oplus \ldots \oplus M$ has a direct summand isomorphic to $R$. Hence $N / J N$ has a summand isomorphic to $R / J$. Hence $x$ acts as a zero divisor on $N / J N=M / J M \oplus \ldots \oplus M / J M$. Hence $x$ acts as a zero divisor on $M / J M$.

Theorem 1. Let $R$ be a ring, $x$ an element of $R$, and I a two-sided ideal of R. Then:

(i) If $I$ is flat as a left R-module, and $x$ is not a left zero-divisor on $R / I$, then $x$ is not a left zero-divisor on $R / I^{n}$ for any $n$.

(ii) If $I$ is a generator of the category of left $R \cdot$ modules, and $x$ is a left zero-divisor on $R / I$, then $x$ is a left zero-divisor on $R / I^{n}$ for all $n$.

(iii) If $R$ is a free associative algebra over a field (and I arbitrary), then for all $n>0, x$ is a zero-divisor in $R / I$ if and only if it is a zero-divisor in $R / I^{n}$.

Proof. (i) and (ii) are proved by induction on n. For (i), apply Lemma 1, with $M=N=R, f=$ left multiplication by $x, M^{\prime}=N^{\prime}=I^{n-1}$, and $J=I$. For (ii), 
apply Lemma 2 with $M=I, J=I^{n-1}$. We get (iii) from (i) and (ii) and the symmetric statements on the right, and the result that in a free associative algebra $R$, any right or left ideal $I$ is free as a (right or left) $R$-module (Cohn [4]).

III. Free subalgebras. Recall from [7] that if $F=K\langle X\rangle$ is a free $K$-algebra, $r \rightarrow r^{\prime}, r \rightarrow r^{\prime \prime} K$-homomorphisms of $F$ into $K$-algebras $R^{\prime}, R^{\prime \prime}$ with kernels $U, V$ respectively and $T=T\left(F ; R^{\prime},{ }_{11} R^{\prime \prime}\right)$ a free $\left(R^{\prime}, R^{\prime \prime}\right)$-bimodule on generators $\delta(x)$ $(x \in X)$, then the map $x \rightarrow\left(\begin{array}{ll}x & x \\ \delta(x) & 0\end{array},\right)$ extends to a homomorphism $\mu: F \rightarrow\left(\begin{array}{ll}R^{\prime \prime} & 0 \\ T & R^{\prime}\end{array}\right)$ with kernel $U V$. The induced map $\delta: F \rightarrow T$ is a derivation. If we consider $T$ as a right $R^{\prime \text { opp }} \otimes R^{\prime \prime}$ bimodule, then we define, for each $x$, partial derivatives $\partial / \partial x: F \rightarrow R^{\text {opp }} \otimes R^{\prime \prime}$ by the formula $\delta(f)=\Sigma_{x \in X} \delta(x)(\partial f / \partial x)$. It is easily verified that if $m \in F$ is a monomial $m=x_{1} x_{2} \cdots x_{n}\left(x_{i} \in X\right)$, then

$$
\frac{\partial m}{\partial x}=\sum_{x_{i}=x}\left(x_{1}^{\prime} \cdots x_{i-1}\right)^{\prime} \otimes\left(x_{i+1} \cdots x_{n}\right)^{\prime \prime} .
$$

Let now $A=R^{\prime}=R^{\prime \prime}=F /[F, F]$, with $[F, F]$ the commutator ideal of $F$. Since $[F, F]^{2}$ is the kernel of $\mu, \mu$ induces an injection $\bar{\mu}: \bar{F}=F /[F, F]^{2} \rightarrow$ $\left(\begin{array}{ll}A & 0 \\ T & A\end{array}\right)$ and $\partial / \partial x$ induces a partial derivative $\bar{\partial} / \bar{\partial} x: \bar{F} \rightarrow A \otimes A$. Each $\bar{\partial} / \bar{\partial} x$, when restricted to $[\bar{F}, \bar{F}]$ is an $A$-bimodule homomorphism. An element $w \in[\bar{F}, \bar{F}]$ is zero if and only if for all $x, \bar{\partial} w / \bar{\partial} x=0$. Let $\nu$ be the natural homomorphism $\nu: A \otimes_{K} A \rightarrow A \otimes_{A} A=A$. For $m_{1}, m_{2} \in \bar{F}$, then

$$
\frac{\bar{\partial}}{\bar{\partial} x}\left(m_{1} m_{2}-m_{2} m_{1}\right)=\frac{\bar{\partial} m_{1}}{\bar{\partial} x} m_{2}^{\prime}-m_{2}^{\prime} \frac{\bar{\partial} m_{1}}{\bar{\partial} x}+\left(m_{1}^{\prime} \frac{\bar{\partial} m_{2}}{\bar{\partial} x}-\frac{\bar{\partial} m_{2}}{\bar{\partial} x} m_{1}^{\prime}\right) \text {. }
$$

Since $\nu$ acts by removing tensor signs, the composed map $(\bar{\partial} / \bar{\partial} x) \nu$ is zero at $m_{1} m_{2}-m_{2} m_{1}$. Since $(\bar{\partial} / \bar{\partial} x)_{\nu}$ is an $A$-bimodule homomorphism on $[\bar{F}, \bar{F}]$ it follows that $(\bar{\partial} / \bar{\partial} x) \nu$ is zero on $[\bar{F}, \bar{F}]$, and thus can be considered as a derivation $d / d x^{0}: A=\bar{F} /[\bar{F}, \bar{F}] \rightarrow A$. It is immediate from (1) that if we write $x^{\prime}$ for the coset $x+[F, F]$, then, $d / d x^{\prime}$ is indeed the usual partial derivative with respect to $x^{\prime}$ in the polynomial ring $A=K\left[X^{\prime}\right]$.

Lemma 3. Let $A=R^{\prime}=R_{1}=\bar{F} /[F, F]$ and $R^{\prime \prime}=R_{d}=F /[F, F]^{d}$. Let $w_{1}, \cdots, w_{n}$ be elements of $F=K\left\langle x_{1}, \cdots, x_{n}\right\rangle=K\langle X\rangle$ sucb that $w_{1}^{\prime}, \cdots, w_{n}^{\prime}$ are algebraically independent in $R_{1}=K\left[X^{\prime}\right]$. Then the Jacobian matrix $\Gamma_{d}=\left(\partial w_{i} / \partial x_{j}\right)$ is regular over $R_{1} \otimes R_{d}$

Proof. We first treat the case $d=1$.

Let $\nu_{*}:\left(A \otimes_{K} A\right)_{*} \rightarrow A_{*}$ be the map induced by $\nu$ on $n \times n$ matrices. Then, by the foregoing discussion, $\Gamma_{1} \nu_{*}=\left(d w_{i}^{\prime} / d x_{j}^{\prime}\right)$, the Jacobian matrix of the elements $w_{i}^{\prime}$ of $A$. The independence of $w_{i}^{\prime}, \cdots, w_{n}^{\prime}$ insures that $\Gamma_{1} \nu_{*}$ is regular in $A_{n^{*}}$. Thus $\Gamma_{1} \nu_{*}$ has a nonzero determinant. Since $\nu$ is a ring homomorphism, 
$\left(\operatorname{det} \Gamma_{1}\right) \nu=\operatorname{det}\left(\Gamma_{1} \nu_{*}\right)$. So $\Gamma_{1}$ also has a nonzero determinant, and, hence, is regular.

Let now $\pi^{d}$ be the natural map $\pi^{d}: R_{1} \otimes_{K} F \rightarrow R_{1} \otimes_{K} R_{d}$, and let $\pi_{*}^{d}$ be the map induced on $n \times n$ matrices. Then the kernel of $\pi^{d}$ is $R_{1} \otimes[F, F]^{d}$, so that $\operatorname{Ker} \pi_{*}^{d}$ is $\left(R_{1} \otimes[F, F]^{d}\right)_{*}=\left(\left(R_{1} \otimes_{K}[F, F]\right)_{*}\right)^{d}$. Now, since $F$ is a free algebra, $[F, F]$ is free as a left module and it follows that $R_{1} \otimes[F, F]$ is free as a left $R_{1} \otimes F$-module (with basis $1 \otimes(F$-basis for $[F, F])$ ). Thus, when we go to $n \times n$ matrices, $\left(R_{1} \otimes[F, F]\right)_{*}$ is free as a left $\left(R_{1} \otimes F\right)_{*}$ module. Now if $\partial_{0} / \partial_{0} x$ are the partial derivatives with values in $R_{1}^{\text {opp }} \otimes F=R_{1} \otimes F$, then $\Gamma_{d}=\left(\partial_{0} w_{i} / \partial_{0} x\right) \pi_{*}^{d}$, $\Gamma_{1}=\left(\partial_{0} w_{i} / \partial_{0} x\right) \pi_{*}^{1}$. We may now apply Theorem 1 with $R=\left(R_{1} \otimes F\right)_{*}$ and $I=\left(R_{1} \otimes[F, F]\right)_{*}$ to deduce from the first part of the proof that since $\Gamma_{1}$ is left regular, so is $\Gamma_{d^{*}}$. The same argument works just as well qua right modules, so $\Gamma_{d}$ is also right regular.

Let now $C_{d}$ be the variety of all $K$-algebras whose commutator ideal is nilpotent of class $d$. (This is the class of all algebras which satisfy the polynomial identity $\Pi_{i=1}^{d}\left[x_{i}, y_{i}\right] z_{i}=0$.) Then $R_{d}=F /[F, F]^{d}$ is a free algebra in this variety, and we say that a subset $W$ of $R_{d}$ freely generates $R_{d}$ if every relation of $W$ is a polynomial identity of $R_{d^{*}}$. We may ask which subalgebras of $R_{d}$ are again $\mathrm{C}_{d}$-free.

Theorem 2. Let $R_{d}=F /[F, F]^{d}$. A subset of $R_{d} \mathcal{C}_{d^{-f r e e l y} \text { generates a }}$ $\mathcal{O}_{d}$-free subalgebra if and only if it is algebraically independent modulo $\left[R_{d}, R_{d}\right]$.

Proof. We first consider the case where $F$ is finitely generated, $F=$ $K\left\langle x_{1}, \ldots, x_{n}\right\rangle$ and $W$ consists of precisely $n$ elements, $w_{1}+[F, F]^{d}, \ldots, w_{n}+$ $[F, F]^{d}$, which are algebraically independent modulo $\left[R_{d}, R_{d}\right]$. From the discussion at the beginning of this section, $R_{d}$ is embedded in the algebra

$$
\left(\begin{array}{ll}
R_{d-1} & 0 \\
T & R_{1}
\end{array}\right)
$$

$T$ a free $\left(R_{1}, R_{d-1}\right)$-bimodule with basis $\delta\left(x_{1}\right), \ldots, \delta\left(x_{n}\right)$, via the map

$$
x_{i}+[F, F]^{d} \rightarrow\left(\begin{array}{cc}
x_{i}+[F, F]^{d-1} & 0 \\
\delta\left(x_{i}\right) & x_{i}+[F, F]
\end{array}\right)
$$

If $w_{i}+[F, F]^{d}$ corresponds to

$$
\left(\begin{array}{cc}
w_{i}+[F, F]^{d-1} & 0 \\
t_{i} & w_{i}+[F, F]
\end{array}\right)
$$


then, considering $T$ as a right $R_{1} \otimes R_{d-1}$ module, $\left(t_{1}, \ldots, t_{n}\right)=\left(\delta\left(x_{1}\right), \ldots, \delta\left(x_{n}\right)\right) \Gamma_{d-1}$. Since, by Lemma $3, \Gamma_{d-1}$ is regular, $\left\{t_{1}, \cdots, t_{n}\right\}$ is again a basis for a free $R_{1} \otimes R_{d-1}$ module. Now, $w_{1}+[F, F], \cdots, w_{n}+[F, F]$ are algebraically independent, so the map $x_{i}+[F, F] \rightarrow w_{i}+[F, F]$ extends to an injection $R_{1} \rightarrow R_{1}$ and we may assume by induction that $x_{i}+[F, F]^{d-1} \rightarrow w_{i}+[F, F]^{d-1}$ also extends to an injection $R_{d-1} \rightarrow R_{d-1}$. The freedom of the $t_{i}$ 's now insures that the map $x_{i}+[F, F]^{d} \rightarrow w_{i}+[F, F]^{d}$ (which extends to an endomorphism of $R_{d}$ by the freedom of $R_{d}$ ) extends to an injection.

Now for the general case. It is clear that for a subset $Z$ of $R_{d}$ to freely generate a $C_{d}$-free subalgebra it is sufficient that every finite subset of $Z$ freely generates a free subalgebra. Consider $\left\{z_{1}, \cdots, z_{n}\right\}$. Then, for some integer $s,\left\{z_{1}, \cdots, z_{n}\right\}$ is in the subalgebra $S$ of $R_{d}$ generated by $x_{1}+[F, F]^{d}, \ldots$, $x_{s}+[F, F]^{d}$. Since $z_{1}+\left[R_{d}, R_{d}\right], \cdots, z_{n}+\left[R_{d}, R_{d}\right]$ are algebraically independent, we can find $z_{n+1}^{\prime}, \cdots, z_{s}^{\prime}$ in $S$ such that $\left\{z_{1}, \cdots, z_{n}, z_{n+1}^{\prime}, \cdots, z_{s}^{\prime}\right\}$ is still algebraically independent modulo $[S, S]$. From the special case above we deduce that $\left\{z_{1}, \cdots, z_{n}, z_{n+1}^{\prime}, \cdots, z_{s}^{\prime}\right\}$, and hence $\left\{z_{1}, \cdots, z_{n}\right\}$, freely generates a free subalgebra.

For the converse, suppose $z_{1}, \cdots, z_{n} \in R_{d}$ are not algebraically independent modulo $\left[R_{d}, R_{d}\right]$. Then there is a nonzero element $p\left(x_{1}, \ldots, x_{n}\right) \notin[F, F]$ such that $p\left(z_{1}, \ldots, z_{n}\right) \in\left[R_{d}, R_{d}\right]$. Thus $p\left(z_{1}, \ldots, z_{n}\right)^{d}=0$. If $z_{1}, \cdots, z_{n}$ freely generate a $C_{d}$-free subalgebra, then $p\left(x_{1}, \cdots, x_{n}\right)^{n}$ is an identity of $R$, a contradiction since all the identities of $R_{d}$ are in the commutator ideal of $F$.

Note the close resemblance between Theorem 2 and the following result of G. Baumslag [2, Theorem 2]:

Let $G$ be a group which is free in the variety $\delta_{d}$ of groups that are solvable of derived length at most $d$. A subset of $G \mathcal{S}_{d}$-freely generates an $\delta_{d}$-free group if and only if it is linearly independent module $[G, G]$, the commutator subgroup of $G$.

In one direction, Theorem 2 can be extended to (absolutely) free algebras.

Theorem 3. Let $W$ be a subset of the free algebra $F=K\langle X\rangle$ wbich is algebra. ically independent modulo $[F, F]$. Then $W$ freely generates a free subalgebra of $F$.

Proof. By extending $W$ if necessary, we may assume that $W$ is indexed by the elements of $X$. Consider the endomorphism $\alpha$ of $F$ defined by $x \rightarrow w_{x}$, and suppose $0 \neq u \in \operatorname{Ker} a$. Choose $d$ such that $u \notin[F, F]^{d}$. (This can be done since $\bigcap_{k=1}^{\infty}[F, F]^{k}=0$.) Then $\alpha$ induces a map $\alpha_{d}: F /[F, F]^{d} \rightarrow F /[F, F]^{d}$ which, by the previous theorem, is injective. However, $\left(u+[F, F]^{d}\right) a_{d}=u a+$ $[F, F]^{d}=0$, so that $u \in[F, F]^{d}$, a contradiction. Thus Ker $\alpha=0$ and we are done.

IV. Lie algebras. In this section, we prove a representation theorem for Lie algebras. 
Throughout what follows, $L$ will be a Lie algebra over a field $K$, and $A$ its universal enveloping algebra. We denote Lie multiplication by $[$,$] , so that it$ agrees with the commutator operation in $A$. We must begin with some results on the relation between $L$ and $A$.

If $B$ is a $K$-basis for $L$, and we totally order $B$, then an ascending monomial will mean an expression $x_{1} x_{2} \cdots x_{n}$ (representing an element of $A$ ), with $n \geq 0$, $x_{i} \in B$, and $x_{1} \leq x_{2} \leq \cdots \leq x_{n}$. By the Poincaré-Birkhoff-Witt theorem, the ascending monomials form a $K$-basis for $A$. There is an explicit reduction procedure for expressing any monomial in $B$ as a $K$-linear combination of ascending monomials (cf. Jacobson [5, Chapter V], also Bergman [3, §4]). If $y=y_{1} y_{2} \cdots y_{m}$ is a monomial and $y_{i}>y_{i+1}$ for some $i$, we write:

$$
\begin{aligned}
y & =y_{1} \cdots y_{i} y_{i+1} \cdots y_{m} \\
& =\left(y_{1} \cdots y_{i+1} y_{i} \cdots y_{m}\right)+\left(y_{1} \cdots\left[y_{i}, y_{i+1}\right] \cdots y_{m}\right),
\end{aligned}
$$

and then express $\left[y_{i}, y_{i+1}\right] \in L$ as a $K$-linear combination of elements of $B$. Thus, $y$ is a linear combination of monomials of smaller length, $m-1$, and a monomial which is closer to being ascending. So this process, applied to the terms of any expression, will eventually terminate in a linear combination of ascending monomials, the "canonical form" for the element in question.

Now suppose $U$ is a Lie ideal in $L$. Let us first choose a $K$-basis $B(U)$ for $U$, then extend this to a basis $B$ of $L$. Suppose a monomial $y$ in $B$ has at least one factor $y_{j}$ belonging to $B(U)$. Then we claim that a reduction (2) takes $y$ to a linear combination of monomials which again have a factor in $B(U)$. Indeed, if $j \neq i, i+1$ in (2), this is trivial, while if $j=i$ or $i+1$, then $\left[y_{i}, y_{i+1}\right]$ again lies in $U$ because $U$ is an ideal, so $\left[y_{i}, y_{i+1}\right]$ is a linear combination of elements of $B(U)$; the assertion is then clear.

Let $U_{A} \subseteq A$ denote $A U A$, the ideal of $A$ generated by $U$. The elements of $U_{A}$ are by definition those that can be written as linear combinations of (arbitrary) monomials having a factor in $B(U)$. It follows from the above observations that the canonical form for an element of $U_{A}$ will still be as a linear combination of such monomials. In particular, since $L$ is the subspace of $A$ spanned by monomials of length 1 , we have $U_{A} \cap L=U$. This can also be seen directly from the Poincaré-Birkhoff-Witt theorem applied to $L / U$, which has universal enveloping algebra $A / U_{A}$. But the analogous result we shall now obtain for two ideals is not so trivial.

Let $U$ and $V$ be two Lie ideals of $L$. Let us construct the basis $B$ for $L$ as follows. First choose a basis $B([U \cap V, U \cap V])$ for the ideal $[U \cap V, U \cap V]$ $\subseteq L$; extend this to a basis $B(U \cap V)$ of the ideal $U \cap V$; extend $B(U \cap V)$ on the one hand to a basis $B(U)$ of $U$, and on the other hand to a basis $B(V)$ of $V$, and note 
that $B(U) \cup B(V)$ will form a basis of the ideal $U+V$ (cf. diagram).

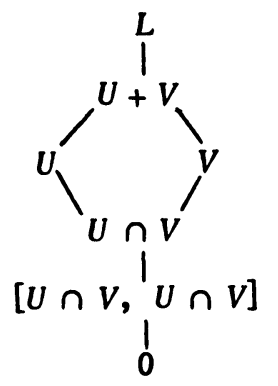

We extend $B(U) \cup B(V)$ to a basis $B=B(L)$ of $L$.

We choose any total ordering of. $B$ subject to the condition that all elements of $B(U)$ not lying in $B(U \cap V)$ should be < all elements of $B(U \cap V)$, which should in turn be $<$ all other elements of $B(V)$.

Now put $U_{A}=A U A$ and $V_{A}=A V A$. We wish to study the ideal $U_{A} V_{A}=$ $A U A V A$. For this purpose, let $S$ denote the set of all monomials on $B$ which either (i) involve an element of $B(U)$ to the left of (i.e., preceding, possibly with some other terms in between) an element of $B(V)$, or (ii) involve an element of $B([U \cap V, U \cap V])$. Clearly every member of $S$, and hence every linear combination of members of $S$ represents an element of $U_{A} V_{A}$, and conversely, $U_{A} V_{A}$ is spanned by the elements represented by monomials in $S$.

We now claim that any reduction of the form (2) will take a monomial $y \in S$ to a linear combination of monomials in $S$. It is clear from earlier observations. that if $y$ satisfies condition (ii), so will all the terms in the expression to which it reduces. Suppose, on the other hand, that $y$ has a term $y_{j} \in B(U)$ to the left of a term $y_{k} \in B(V)$ (i.e., $j<k$ ). If neither $j$ nor $k$ belongs to $\{i, i+1\}$ (in the notation of (2)), the result is trivial, and if only one of them does, it is straightforward, like our earlier result. So suppose, finally, that $j=i, k=i+1$. In order for the reduction (2) to be applicable, the element $y_{j} \in B(U)$ must be greater, under our ordering, than $y_{k} \in B(V)$. By the conditions on our ordering, this can only happen if $y_{j}$ and $y_{k}$ both lie in $B(U \cap V)$. In this case, when we apply (2) the term of length $m$ resulting contains the sequence $y_{k} y_{j}$, and hence still has a member of $B(U)$ (actually a member of $B(U \cap V)$ ) to the left of a member of $B(V)$ (ditto), while $\left[y_{j}, y_{k}\right] \in[U \cap V, U \cap V]$, so the monomials of length $m-1$ all involve terms in $B([U \cap V, U \cap V])$.

It follows that $U_{A} V_{A}$ consists of those elements whose canonical representation involves only monomials from $S$. Intersecting with $L$, spanned by the monomials of length 1 , we get:

Theorem 4. Let $U, V$ be ideals of the Lie algebra $L$. Let $A$ be the universal 
associative enveloping algebra of $L$ and let $U_{A}=A U A, V_{A}=A V A$ be the ideals generated by $U$ and $V$ respectively in $A$. Then $U_{A} V_{A} \cap L=[U \cap V, U \cap V]$. $\square$

Note that the improper ideal $L$ of $L$ induces the augmentation ideal $J=$ $L_{A} \subseteq A$.

Corollary. If $U$ is an ideal of $L$, and $J$ the augmentation ideal of $A$, then, $[U, U]=U_{A} J \cap L=U_{A}^{2} \cap L=J U_{A} \cap L$. $\square$

If $L$ is the free Lie algebra on the set $X$, then $A=K\langle X\rangle=F$ is free associative. So using Theorem 1 of [7] we obtain:

Theorem 5. Let $L$ be a free Lie algebra on the set $X, L^{\prime}, L^{\prime \prime}$ I.ie algebras, $l \rightarrow l^{\prime}, l \rightarrow l^{\prime \prime}$ maps of $L^{\text {into }} L^{\prime}$ and $L^{\prime \prime}$ with kernels $U, V$ respectively, $R^{\prime}$, $R^{\prime \prime}$ the universal enveloping algebras of $L^{\prime}, L^{\prime \prime}$ and $T$ a free $\left(R^{\prime}, R^{\prime \prime}\right)$-module generated by $\{\delta(x) ; x \in X\}$ Then the kernel of the map

is $[U \cap V, U \cap V]$.

$$
L \rightarrow\left(\begin{array}{cc}
L^{\prime \prime} & 0 \\
T & L^{\prime}
\end{array}\right) \text { defined by } x \rightarrow\left(\begin{array}{cc}
x^{\prime \prime} & 0 \\
\delta(x) & x^{\prime}
\end{array}\right)
$$

Proof. By Theorem 1 of [7],

$$
x \rightarrow\left(\begin{array}{cc}
x^{\prime \prime} & 0 \\
\delta(x) & x^{\prime}
\end{array}\right) \text { induces a map } K\langle X\rangle \rightarrow\left(\begin{array}{ll}
R^{\prime \prime} & 0 \\
T & R^{\prime}
\end{array}\right)
$$

whose kemel is $U_{F} V_{F}$. The result follows immediately.

In particular with $L^{\prime \prime}=0$, we find from the corollary that the map

$$
\left.x \rightarrow\left(\begin{array}{cc}
0 & 0 \\
\delta(x) & x^{\prime}
\end{array}\right) \text { defines an embedding } L / U U, U\right] \rightarrow\left(\begin{array}{ll}
0 & 0 \\
T & L^{\prime}
\end{array}\right) .
$$

If $U$ is an ideal of $L$, and $R^{\prime}$ is the universal enveloping algebra of $L / U$, then the adjoint representation of $L$ induces an $R^{\prime}$-module structure on $U /[U, U]$. If $J$ is the augmentation ideal of the free algebra $F=K\langle X\rangle$, then the injection $U \rightarrow J$ induces an $R^{\prime}$-module homomorphism $U /[U, U] \rightarrow J / U_{F} J$. The corollary shows that this map is injective. (This was shown by Labute [G] if $U$ is generated by a single element.)

If $L$ is finitely generated and $R^{\prime}$ is left Noetherian, then $J / U_{F} J$ is left Noetherian and hence so is $U /[U, U]$. This can be used to give an alternate proof of a recent theorem of Amayo and Stewart [1]:

Let $L$ be a finitely generated Lie algebra baving an Abelian ideal $U$ sucb that the universal enveloping algebra of $L / U$ is left Noetherian. Then $L$ satisfies the maximal condition for ideals. 


\section{REFERENCES}

1. R. Amayo and I. Stewart, Finitely generated Lie algebras, J. London Math. Soc. (2) 5 (1972), 697-703.

2. G. Baumslag, Some subgroup theorems for free vegroups, Trans. Amer. Math. Soc. 108 (1963), 516-525. MR 27 \# 4862.

3. G. Bergman, The diamond lemma for ring theory (to appear).

4. P. M. Cohn, On a generalization of the Euclidean algorithm, Proc. Cambridge Philos Soc. 57 (1961), 18-30. MR 22 \#9514; 22, 2547.

5. N. J acobson, Lie algebras, Interscience Tracts in Pure and Appl. Math., no. 10, Interscience, New York, 1962. MR $26 \# 1345$.

6. J. Labute, Algèbres de Lie et pro-p-groupes définis par une seule relation, Invent. Math. 4 (1967), 142-158. MR 36 \#1581.

7. J. Lewin, A matrix representation for associative algebras. I, Trans. Amer. Math. Soc. 188 (1974), 293-308.

DEPARTMENT OF MATHEMATICS, SYRACUSE UNIVERSITY, SYRACUSE, NEW YORK 13210 